\title{
15
}

\section{An Explorative Model for Federated Trading in Distributed Computing Environments}

\author{
Ok-Ki Lee and Steve Benford \\ Communications Research Group, Department of Computer Science; University of Nottingham, \\ Nottingham, NG7 2RD, United Kingdom
}

We propose a model for trading in very large-scale distributed computing environments which is based on the gradual evolution of a federated trading space through a process of continual exploration and evaluation, rather than on the imposition of a strictly managed hierarchy. In our model, each trader autonomously acquires local knowledge of the trading space, called trading knowledge, through a process of distributed resource discovery. Trading knowledge typically consists of trader links which reference other traders. Trader links also contain a measure of affinity: a strength of attraction based on a comparison of so called service and interest profiles, perhaps combined with a history of how useful and reliable other traders have proved to be. This notion of affinity helps a trader to decide how to resolve import requests which cannot be satisfied locally. It also helps a trader to decide which trader links to retain as it periodically and autonomously explores the trading environment. Instead of being concerned with the detailed management of individual trader links, human managers can then control the evolution of the trading space through a number of high level management policies. These include service and interest profiles, definitions of affinity and instructions on when and how exploration should occur. Our paper also describes a reference implementation of the model within the ANSAware distributed processing environment called the Explorative Trading Service (ETS).

Keyword Codes: C.2.4; H.3.3

Keywords: Distributed Systems, Federated Trading, Resource Discovery

\section{INTRODUCTION}

As the number of computer systems involved in distributed computing increases, one centralised trader will simply not be enough to accommodate a large number of exporters and importers. It therefore becomes necessary that trading be performed in a distributed manner. However, in the research community it has been an issue how to organise and manage such a distributed trading service [ANSA 89, ISO Trader 92, Maffeis 93, Linden 92].

One proposed approach has been to use global naming services such as the X.500 Directory [ISO DS 88] to support distributed trading [ISO Trader 92]. This approach aims to establish a global naming/trading hierarchy with traders being linked through the naming service. Global naming hierarchies are intended to simplify query resolution, ease administration and are also claimed to be representative of the underlying structure of human organisations. However, along with others [Linden 92], we have grave doubts about the feasibility of the hierarchical approach: 
- Although hierarchy is technically appealing due to its relative simplicity to implement in software, it is politically difficult to implement in practice as it requires the establishment of powerful, maybe global administrative authorities. Such authorities may be particularly hard to establish in a climate which favours deregulation of telecommunication services.

- It is not at all clear that organisations are hierarchies. Various, more recent, models have been proposed for human organisational structures including organisations as networks, matrices and organisms.

- Hierarchical information structures tend to be unwieldy and resistant to change. Indeed, one can argue that this is why the hierarchical database model has generally been superseded by the relational and object oriented models. It was an early assumption of X.500 that the top levels of the global hierarchy would be stable. However, a brief look at international events since 1988 (the date of the first X.500 standard) show this to be generally untrue.

For these reasons, we propose to adopt a more ad-hoc and explorative approach to organising a federated trading service. In particular, we propose that a trading space should grow and evolve through a process of continual exploration and that each trader should autonomously maintain a personalised view of the trading space. This approach has been motivated by the observation that the real world is not necessarily well-organised and often supports autonomy and ad-hoc organisation [Saltzer 1978]. We have also been greatly influenced by the recent success of a number of NIR (Network Information Retrieval) projects, particularly the work of Netfind [Schwartz \& Tsirigotis 91], Internet Gopher [McCahill 92] and Prospero [Neuman 92]. We suspect that their rapid spread may be at least partly due to the autonomous and de-centralised nature of their organisation.

Section two of our paper clarifies the design goals behind our model. Section three then describes the model in detail. Finally section four describes a reference implementation of the model in the ANSAware [ANSA 92] environment.

\section{DESIGN GOALS}

The design goals of any distributed trading service might include the following:

- Partitioning responsibility - responsibility for managing many different services may be divided between different traders.

- Fault tolerance through redundancy - replicating multiple instances of a service across multiple traders may enable fault tolerance in the light of both service and trader failures.

- Decentralised ownership - services will be provided, owned and managed by different organisations. These organisations may wish to retain autonomous management of their own services, even when they cooperate with each other, let alone when they compete.

In addition to these general goals, the development of our trading model has been driven by a number of more specific requirements.

- Realistic assessment of others - when a service is requested but not available locally, a trader might rely on other traders to find it on its behalf (the basic idea behind distributed trading). However, the issue of trustworthiness then arises. In the real world, it is not always the case that organisations or people actually provide the service or quality of service that they claim to. Why should it be any more true of their traders? Realistic 
appraisal of the claims of other traders should be a part of distributed trading. In other words, we should support traders in distinguishing "good" friends from "bad" ones. Furthermore, this process might involve some notion of learning from past experience (for example, lookup success rate, actual quality of service delivered and availability).

- Explore, adapt and evolve - our model should not assume a consistent or stable environment. Instead, in a large system, services and traders will constantly appear, change and disappear. Such changes cannot be instantly conveyed to all interested parties. Traders should be therefore be supported in exploring the trading space, interrogating each other about services offered and other traders known about. Furthermore, they should be prepared for inconsistencies in trading knowledge and must be able to adapt. The overall picture of the trading environment will therefore be one of a constantly evolving system with no one trader ever understanding the complete picture. Furthermore, the ability for traders to cope and adapt quickly will be critical to their success.

- Ease of human administration - As seen in NIR, human administrators are required to spend a significant amount of time maintaining their servers. In our model, human administrators should only be required to specify high level management policies. The traders themselves should then take care of administrative details as much as possible.

Having clarified the design goals behind our model, the following section now introduces the model itself in terms of its key concepts.

\section{THE EXPLORATIVE MODEL FOR FEDERATED TRADING}

Our model assumes as its starting point the existing concepts of service providers, service consumers and traders. On top of these we define the concepts of trader knowledge, trader links, distributed request resolution, trader knowledge management, affinity, service, interest and reputation profiles, exploration, announcement and management policies.

\subsection{Trader links and trader knowledge}

We conceive of a distributed trading system as involving two logical planes, a service plane in which service providers and consumers exist and a trading plane in which multiple traders exist. As a result of trading, the service consumers and providers become connected by service links, thus enabling the distribution of applications. In turn, the traders are connected by trader links, thus enabling the distribution of the trading process itself. This structure is shown in figure 1.

A trader link tells a local trader how to connect to a given remote trader. It therefore defines a unidirectional ordered pair of traders. In order to achieve its purpose a trader link must contain at least minimal addressing information (it may contain other information as we shall see below). For example, it might take the form of a Uniform Resource Locator (URL) [Berners-Lee]. In a very large and dynamic distributed system, it is unlikely that a trader will be able maintain links to all other traders. Instead, each trader will utilise a local pool of trading links called its trader knowledge. Consequently, the general structure of trading space is that of a directed graph. 


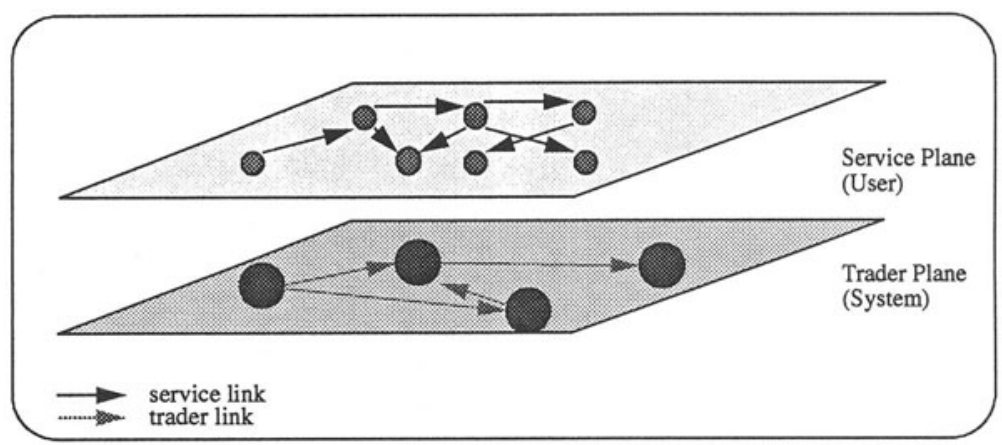

Figure 1: The trading universe

\subsection{Distributed request resolution}

Distributed request resolution refers to the way in which a group of traders resolves a service import request. In our view, this process should be as transparent as possible to the service providers and consumers. In other words, a service provider should only be required to contact one trader in order to resolve an import request.

When an importer makes a request to a trader for a service, the initial trader contacted will first search its local trading space. If the service is found, the trader will of course return the reference (address) of the service to the importer. However, if no local service is found, then the trader will contact its adjacent traders by following its local trader links. These adjacent traders will then search their local trading spaces. If the service is still not found, the initial trader may interrogate its adjacent traders to find out about their links to others and may follow these in search of the service. This process continues until either the service is found or there is good reason to cease (e.g. exceeding a cost/time limit or running out of unexplored trader links).

Notice that we make the local trader responsible for following all trader links and do not adopt a policy of "chaining" whereby requests are recursively passed along a chain of traders via their trader links. We believe that this allows the local trader to retain tighter control over the resolution process, thereby avoiding problems with loops caused by cycles in the trading space and also makes it easier to realise constraints such as time-outs and cost limits.

\subsection{Trader knowledge management}

Trader knowledge management describes the process by which trader links are created, maintained and destroyed over time. A basic approach to this problem might be to provide human administrators with a protocol and management tool for maintaining individual links. However, such fine grained management would impose a potentially large overhead on human managers and leaves unanswered the question of how these managers find out about other traders in the first place. Instead, our model makes the traders themselves responsible for locating other traders of interest and for evaluating how useful they might be. This is achieved through a process of exploration. The traders themselves might then be responsible for maintaining individual links and human managers could be provided with a set of higher level management policies for 
controlling the exploration process. Thus, the trader knowledge management problem involves a number of sub-issues:

- Basic mechanisms for traders to manage trader links (e.g. establish, release, propagate);

- Techniques for assessing the usefulness of other traders;

- Mechanisms to support automated exploring;

- Definition of suitably high level management policies for humans.

\subsection{Affinity}

Associated with each trader link is a notion of affinity. The affinity of a link is intended as a measure of how useful the local trader believes the remote trader to be. In the simplest case, affinity might be expressed as a numeric value normalised to be in some range (e.g. between 0 and 1) which expresses the degree of overlap between the interests of the local trader and the services supported by the remote trader (more of this later). Thus, the trading space now becomes a weighted directed graph. Figure 2 shows a snapshot of a simple trading space. In this example we see that the trader $\mathrm{Tf}$ currently has trader links with four other traders, $\mathrm{Th}, \mathrm{Ti}, \mathrm{Tj}$ and $\mathrm{Td}$ with affinities ranging from 0.9 to 0.7 .

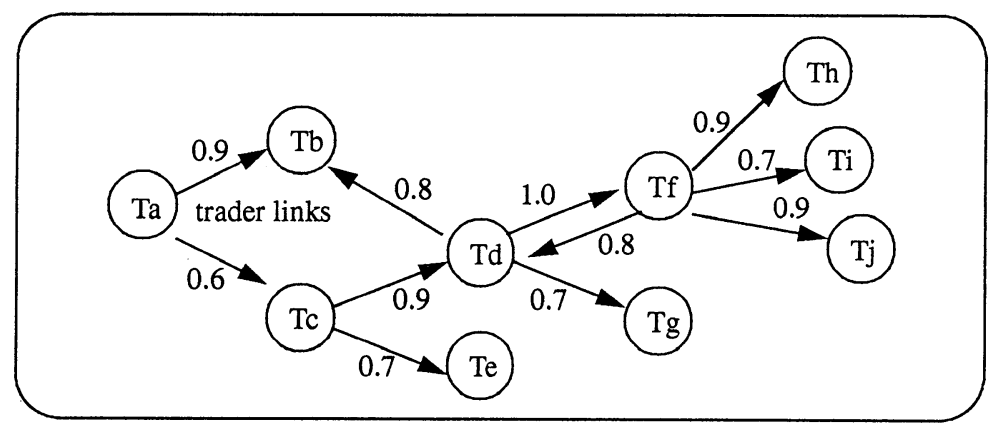

Figure 2: An example trading space with affinity

Affinity plays two roles in our model. First, it provides a way for a trader to decide whether or not to retain an on-going link to another trader. For example, a trader might choose to remember all links whose affinity exceeds some threshold. Alternatively, if a trader can only store a limited number of links (perhaps for reasons of space or speed of searching) it might compare the affinity of a newly discovered link with its least useful current link in order to decide which of the two to keep. Second, affinity may enhance the process of distributed request resolution by allowing a trader to prioritise the order in which it contacts other traders. The assumption here is that a high affinity link offers the best chance of a quick resolution.

\subsection{Service and interest profiles}

The concept of affinity requires that a trader can assess how the services offered by another trader match its own interests. This idea is more formally captured in the concepts of service profile and interest profile. A service profile is a description of the services which one trader is 
prepared to offer to others (note that a trader may not be prepared to offer all of its services). Service profiles might range from simple lists of service types to more complex descriptions (e.g. involving offers of quality of service). An interest profile describes those services which a trader is interested in finding in other traders and may be described in the same way as the service profile (in this case quality of service would refer to desired quality). Interest profiles might be automatically generated from logs of clients' import requests over a period of time. Similarly, service profiles might automatically be generated from interface specification code (e.g. from IDL in ANSA).

The degree of difference between a single trader's service and interest profiles will reflect is motivation for entering into distributed trading. A strong overlap suggests that fault tolerance through redundancy is of prime importance whereas a weak overlap suggests a partitioning responsibility for managing different services.

The affinity that one trader has for another is then a function of the interest profile of the former and the service profile of the latter. The exact nature of such an affinity function will of course depend on the nature of the profiles. For example, were the profiles to be simple lists of services then a basic function expressing the affinity of trader A for trader B might be the size of the intersection of the interest profile of $A$ and service profile of $B$ as a fraction of the interest profile of A.This delivers the value 0 when B provides none of A's desired services and 1 when it provides them all. However, many other functions might be possible. In fact, we might usefully draw on techniques from the domain of information retrieval systems when constructing such functions. (e.g. we might apply weighting factors to the different entries in service and interest profiles).

\subsection{Reputation profile}

Affinity as described above compares the stated interests of one trader against the promises of another. We argue that it is important that this be tempered by a trader's actual experience. For example, if a remote trader continually fails to resolve requests, the local trader might reduce its affinity for it. Conversely, a low affinity link that continually delivers useful results might increase in strength. The quality and reliability of the actual services found should also modify affinity. For example, if the actual quality of service encountered is frequently worse than that promised, affinity might drop. In other words, the affinity associated with a given link should be dynamically modified by a trader's actual experiences both in terms of successful resolution of requests and quality of end services located. Thus, a trader can learn by experience. These ideas are captured in the concept of a reputation profile, a collection of statistical information gleaned through the process of interacting with other traders and which serves as a measure of the reputations of these other traders in the eyes of the local trader.

\subsection{Exploration and announcement}

So far we have introduced concepts to allow a trader to adopt an informed view of other traders it encounters. However, how does it find out which other traders are out there in the first place?

Our model supports this discovery through the processes of exploration and announcement. Many of our ideas here have been strongly influenced by work on resource discovery within the Network Information Retrieval (NIR) community.

Exploration involves a trader in periodically navigating the trading space by following trader 
links. This process is similar to distributed request resolution as described earlier, in that a trader follows its links to other traders and then follows their links to yet others. For each trader encountered, it invokes its affinity function. At the end of the exploration the trader then invokes some local decision procedure in order to update its trader knowledge (e.g. deciding which links to keep and which to drop).

The obvious similarity between distributed request resolution and exploration relate to a distinction between on-demand and off-demand exploration.

- On-demand exploration means that a trader starts exploring when a service is requested and the service is not found locally. In other words, it refers to a process of distributed request resolution which might also include evaluation of other traders en route.

- Off-demand exploring means that a trader explores automatically as a separate process, probably during periods when other activity is low (e.g. nightly).

In contrast to exploration, announcement involves traders who are involved in some change actively spreading this news. Typical changes might involve the creation of new traders, the removal of existing ones or significant changes in service profile. Announcements, which may include revised service profile and addressing information, are then passed from trader to trader over a special protocol using trader links. Thus, announcement is the converse of exploration.

It might also be useful to combine exploration and announcement into specialised exploration agents whose job it is to autonomously explore the trading space, collate results and announce them in some digested form (similar to Robots and Spiders in NIR systems [Koster]).

\subsection{Management policies}

Many different aspects of our model might be parameterised in order to control the manner in which the distributed trading process works. These include: controlling distributed request resolution (e.g. by specifying time or cost limits); defining service and interest profiles; defining affinity functions; constraining the amount of local trader knowledge to be held at any one time; defining decision procedures; defining reputation profiles; and configuring the exploration and announcement processes (e.g. saying when and how often they should occur and how far they should spread).

Between them, these parameters represent a set of management policies that allow human managers to influence the trading process and configure local traders without having to manipulate individual trader links. These 'high-level' policies might then be stored in the local trader and be created and updated through a trader management client using a specialised protocol.

This concludes our presentation of the basic model. The following section describes how we have implemented it in the ANSAware environment.

\section{REFERENCE IMPLEMENTATION}

This section describes a reference implementation of our model called the Explorative Trading Service (ETS). Our aim here has been to demonstrate how the model can be realised in a typical ODP environment and to explore the components and interfaces required to support it. Future work will involve using our implementation to test the model through large scale simulation experiments. Our reference implementation has been developed on top of the ANSAware 4.0 platform. The implementation environment consists of a collection of SUN $3 \mathrm{~s}$ and 
SUN 4s which run ANSAware 4.0 under SunOS 4.1.3. The languages to be used are C, $\mathrm{C}^{++}$and $\mathrm{Tcl} / \mathrm{Tk}$ [Ousterhout 93].

ETS consists of several components: the Explorative Trader (ET), Trader Browser, Trader Manager and Interface Repository. These are shown in figure 3 along with a summary of the interfaces they offer to other components. Full specification of these components is beyond the scope of this paper, but can be obtained from the authors on request. Instead, the following paragraphs briefly summarise each in turn.

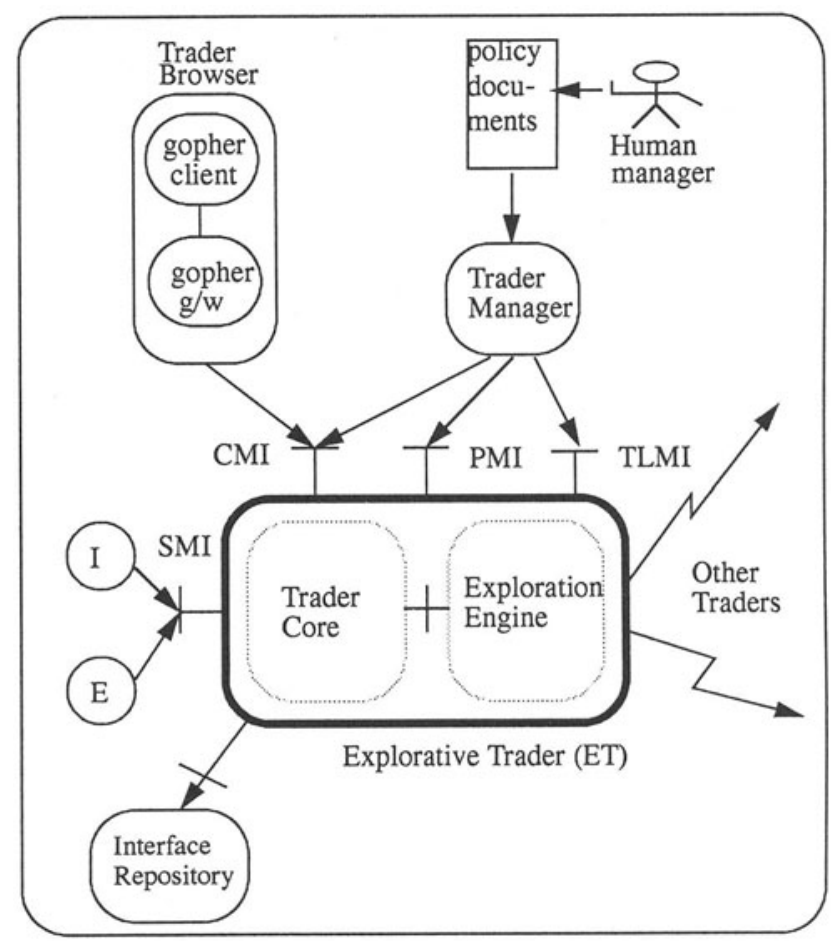

Figure 3: ETS Architecture

\subsection{Explorative trader}

The Explorative Trader is the main component of ETS and consists of two further subcomponents: the trader core which provides a standard localised trading service and the exploration engine which is responsible for distributed request resolution and trader knowledge management. ET provides four interfaces to other components:

- Service Management Interface (SMI) - defines operations which can be directly used by an application (i.e. a collection of importers and exporters). An importer and an exporter make use of this interface to import and export a service. 
- Context Management Interface (CMI) - is used to manage internal organisation of a trader. Specifically it provides operations for managing trader contexts.

- Trader Link Management Interface (TLMI) - consists of operations which are related to trader link management. It supports trading in exploring the trading space and in establishing links to other traders. The intention here is that, via a process of exploration, traders can acquire knowledge of other traders and so engage in 'federated trading'. The interface plays a key role in our exploration model for trading.

- Policy Management Interface (PMI) - provides operations for imposing policies on a trader. This interface will affect the static and dynamic behaviours of a trader. Categorically, the interface supports profile management policy and trader link management policy. For example, the trader manager makes use of this interface to change the exploration time-out or depth of search etc.

\subsection{Trader browser}

The Trader Browser allows a human manager to browse and explore the local trading space within a specified trader via the Context Management Interface. It has been our intention to re-use existing tools for ETS wherever possible. In particular, the Internet Gopher [McCahill 92] has attracted our attention, because its protocol is relatively simple and there already exist a number of popular user interfaces such as xgopher or Mosaic available across a variety of platforms. Consequently, we have developed a Gopher to Trader gateway, which allows any gopher client to browse the trading space. It will be extended to support service and link level operations in the near future.

\subsection{Trader manager}

The trader manager is an ETS component which allows a human administrator or an application program to manage the Explorative Trader via a set of given interfaces. Mainly, the trader manager is involved in the following:

- Manual trader link management when it is required

- Providing trader link management policy for ET - for example, maximum number of trader links to explore, when to start/stop exploring

- Managing trader contexts inside ET

\subsection{Interface repository}

ETS includes an interface repository which is mainly intended to store IDL definitions. As an example service, we have developed a dictionary interface ${ }^{1}$ which supports the following operations: lookup, synonym and antonym.

\section{SUMMARY}

Our paper has been concerned with an explorative approach to distributed trading. Our main design goals have been to support autonomy, decentralisation, learning by experience, gradual evolution of a trading space, realistic assessment of other trader's capabilities and high-level

1. In fact, an ANSAware "wrapper" is used to encapsulate an existing dictionary service on the network. 
human administration. These goals are reflected in our explorative trading model which is summarised by figure 4 .

The figure shows that trader space takes the form of a weighted directed graph consisting of traders connected by trader links. A trader link contains some addressing information as well as a measure of affinity derived by comparing the interest profile of a local trader with the service profile of a remote one. Trader links are used as part of distributed request resolution where a local trader coordinates interaction with traders to which it is linked (and perhaps other ones to which they are linked) until a request is resolved or terminated. Trading knowledge is acquired and updated through the processes of exploration and announcement, influenced by the concepts of affinity and also reputation, the latter allowing a trader to learn from experience. Finally, humans manage the system through a set of high level management policies which include the profiles mentioned above as well as policies for controlling how and when exploration and announcement take place.

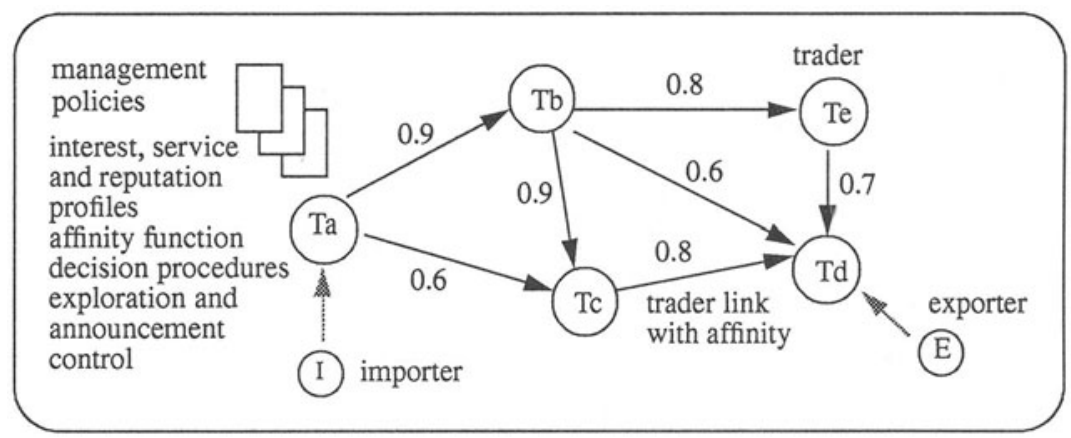

Figure 4: Summary of the model

Finally we have described a reference implementation of the model called the Explorative Trading Service (ETS), based on ANSAware. ETS shows some of the components and interfaces which may be required to support the model in future large scale distributed systems.

\section{REFERENCES}

[ANSA 89] The ANSA Reference Manual, Architecture Projects Management Ltd., Cambridge, UK, 1989.

[ANSA 92] ANSAware 4.0: Application Programming in ANSAware, Architecture Projects Management Ltd., March 1992.

[Berners-Lee] Tim Berners-Lee, Uniform Resource Locators, URL: ftp://info.cern.ch/pub/ietf/url4.ps,(.txt).

[ISO DS 88] ISO and CCITT, Information Processing Systems - Open Systems Interconnection - The Directory, ISO 9594-108, CCITT X.500-X.521, 1988.

[ISO Trader 92] Working Document on Topic 9.1- ODP Trader, ISO/IEC JTC1/SC21/WG7/N743, November, 1992.

[Koster] Martijn Koster, List of Robots, URL: http://web.nexor.co.uk/mak/doc/robots/active.html.

[Maffeis 93] S. Maffeis, Electra - Making Distributed Programs Object-Oriented, Proceedings of the Symposium on 
Experiences with Distributed and Multiprocessor Systems IV, USENIX, San Diego, CA, USA, September 1993.

[McCahill 92] M. McCahil, Internet Gopher: A Distributed Server Information System, ConneXions - The Interoperability Report, 6(7), pp. 10-14, Interop Inc., July 1992.

[Neuman 92] B. C. Neuman, Prospero: A Tool for Organizing Internet Resources, Electronic Networking: Research, Applications, and Policy, 2(1), pp. 30-37, Meckler Publications, Wesport, CT, USA, Spring 1992.

[Linden 92] R. J. van der Linden, Naming and Federation, in Naming Facilities in Internet Environments and Distributed Systems (Special Issue of the Distributed Processing Technical Committee Newsletter), Bharat Bhargava (ed.), Volume 14, Number 1, pp 21-27, IEEE Technical Committee on Distributed Processing, June 1992.

[Ousterhout 93] John Ousterhout, An Introduction to Tcl and Tk, Addison-Wesley, 1993.

[Saltzer 78] J. H. Saltzer, Research Problems of Decentralized Systems with Largely Autonomous Nodes, in R. Bayer, R.M. Graham and G. Seemuller (eds.) Operating Systems: An Advanced Course, Lecture Notes in Computer Science 60 , pp. 583-593, Springer-Verlag, 1978.

[Schwartz \& Tsirigotis 91] M. F. Schwartz and P. G. Tsirigotis, Experience with a Semantically Cognizant Internet White Pages Directory Tool, J. Intenetworking: Research and Experience, 2(1), pp. 23-50, March 1991. 